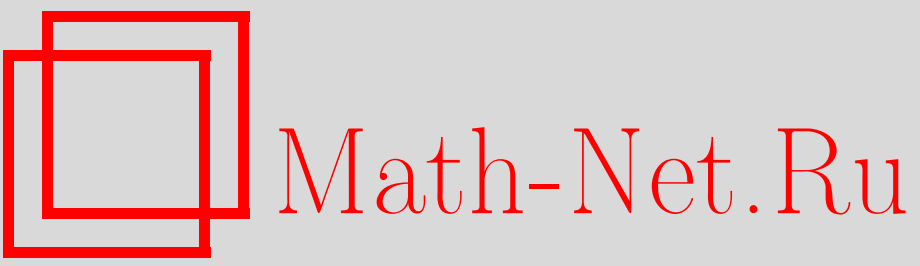

М. П. Бурлаков, В. М. Бурлаков, Бесконечные произведения биномов и разбиения чисел, Итоги науки и техн. Сер. Соврем. мат. и ее прил. Темат. обз., 2020, том $181,9-15$

DOI: https://doi.org/10.36535/0233-6723-2020-181-9-15

Использование Общероссийского математического портала Math-Net.Ru подразумевает, что вы прочитали и согласны с пользовательским соглашением

http://www. mathnet.ru/rus/agreement

Параметры загрузки:

IP : 3.85 .5 .30

26 апреля 2023 г., 16:20:08 


\title{
БЕСКОНЕЧНЫЕ ПРОИЗВЕДЕНИЯ БИНОМОВ И РАЗБИЕНИЯ ЧИСЕЛ
}

\author{
(C) 2020 г. $\quad$ М. П. БУРЛАКОВ, В. М. БУРЛАКОВ
}

\begin{abstract}
АннотАция. В статье рассматриваются разложения функций в бесконечные произведения степенных биномов. Приведены также формула для представления экспоненты такими произведениями и вывод формул для вычисления количества разбиений натуральных чисел на слагаемые.
\end{abstract}

Ключевые слова: бесконечное произведение, бесконечная система линейных уравнений, разбиение числа.

\section{INFINITE PRODUCTS OF BINOMIALS AND PARTITIONS OF NUMBERS}

\author{
(c) 2020 M. P. BURLAKOV, V. M. BURLAKOV
}

\begin{abstract}
In this paper, we consider expansions of functions into infinite products of power binomials. We also propose a formula for representing the exponential function by such products and formulas for calculating the number of partitions of natural numbers.
\end{abstract}

Keywords and phrases: infinite product, infinite system of linear equations, partition of a number.

AMS Subject Classification: 05E06, 11A06

Леонард Эйлер в своей знаменитой работе «Введение в анализ бесконечных» показал, что произведения степенных биномов связаны с количеством различных разбиений натуральных чисел на слагаемые (см. [5]). В частности, в тождествах

$$
\prod_{n=1}^{\infty}\left(1-x^{n}\right)^{-1}=1+\sum_{n=1}^{\infty} L(n) x^{n} \text { и } \prod_{n=1}^{\infty}\left(1+x^{n}\right)=1+\sum_{n=1}^{\infty} l(n) x^{n},
$$

функция натурального аргумента $L(n)$ дает количество неупорядоченных представлений числа $n \in \mathbb{N}$ в виде суммы равных или неравных натуральных слагаемых, а значения функции натурального аргумента $l(n)$ - это количества неупорядоченных представлений числа $n \in \mathbb{N}$ в виде суммы неравных натуральных слагаемых (см. [4]). Эти примеры показывают пользу изучения бесконечных произведений степенных биномов для решения задач в разных областях математики.

Обобщая произведения, приведенные в тождествах (1), поставим задачу представления функций бесконечными произведениями степенных биномов следующего вида:

$$
\prod_{n=1}^{\infty}\left(1-x^{n}\right)^{a_{n}} \quad \text { и } \quad \prod_{n=1}^{\infty}\left(1+x^{n}\right)^{b_{n}}
$$

которые будем называть мультипликативными агрегатами (или просто агрегатами) соответственно первого и второго рода (см. $[1,2])$, а показатели степени $a_{n}$ и $b_{n}$ - их коэффициентами. 
1. Рассмотрим произвольную гладкую функцию $f(x)$, для которой $f(0)=1$, и попробуем найти коэффициенты $a_{n}$ ее представления агрегатом первого рода. Пусть

$$
f(x)=\prod_{n=1}^{\infty}\left(1-x^{n}\right)^{a_{n}} .
$$

Прологарифмировав и продифференцировав тождество (2), получим следующее соотношение:

$$
-\frac{f^{\prime}(x)}{f(x)}=\sum_{n=1}^{\infty} \frac{n a_{n} x^{n-1}}{1-x^{n}}=\sum_{n=1}^{\infty} n a_{n} x^{n-1}\left(\sum_{k=0}^{\infty} x^{n k}\right) .
$$

Если теперь представить отношение $-f^{\prime}(x) / f(x)$ рядом Маклорена, положив

$$
-\frac{f^{\prime}(x)}{f(x)}=\sum_{n=0}^{\infty} q_{n} x^{n}
$$

то из (3) получим бесконечную систему линейных уравнений для определения коэффициентов $a_{n}$. Используя суммирование по делителям числа $n$, можно записать эту систему в следующем виде:

$$
\sum_{m \backslash n} m a_{m}=q_{n-1}
$$

Начало этой системы в явном виде выглядит следующим образом:

$$
\left\{\begin{aligned}
a_{1} & =q_{0}, & a_{1}+7 a_{7} & =q_{6}, \\
a_{1}+2 a_{2} & =q_{1}, & a_{1}+2 a_{2}+4 a_{4}+8 a_{8} & =q_{7}, \\
a_{1}+3 a_{3} & =q_{2}, & a_{1}+3 a_{3}+9 a_{9} & =q_{8}, \\
a_{1}+2 a_{2}+4 a_{4} & =q_{3}, & a_{1}+2 a_{2}+5 a_{5}+10 a_{1} 0 & =q_{9}, \\
a_{1}+5 a_{5} & =q_{4}, & a_{1}+11 a_{11} & =q_{10}, \\
a_{1}+2 a_{2}+3 a_{3}+6 a_{6} & =q_{5}, & a_{1}+2 a_{2}+3 a_{3}+4 a_{4}+6 a_{6}+12 a_{12} & =q_{11}, \quad \ldots
\end{aligned}\right.
$$

Для примера представления функции агрегатом первого рода возьмем экспоненту $f(x)=e^{x}$, для которой $-f^{\prime}(x) / f(x)=-1$. Подставляя $q_{0}=1$ и $q_{1}=q_{2}=\ldots=0$ в систему (5), получим следующую замечательную формулу (см. [2]):

$$
e^{x}=\prod_{n=1}^{\infty}\left(1-x^{n}\right)^{-\mu(n) / n},
$$

где $\mu(n)$ - теоретико-числовая функция Мебиуса (см. [3]).

Система (5) - это треугольная бесконечная система линейных уравнений, все частичные определители которой отличны от нуля. Поэтому система (5) всегда имеет единственное решение. Однако найти это решение в общем виде бывает затруднительно, поскольку для коэффициентов уравнений системы (5) нет явного выражения.

Чтобы исправить эту ситуацию, введем в рассмотрение так называемые мигающие функции натурального аргумента

$$
\beta_{m}(n)=\left\{\begin{array}{ll}
1, & \text { если } n=k m, \\
0, & \text { если } n \neq k m .
\end{array} .\right.
$$

Нетрудно заметить, что систему (5) можно переписать в виде

$$
\sum_{n=1}^{m} n a_{n} \beta_{n}(m)=q_{m-1} .
$$


Эту систему мы будем называть определяющей системой для мультипликативных агрегатов первого рода. Начало этой системы имеет вид

$$
\left\{\begin{aligned}
a_{1} \beta_{1}(1) & =q_{0}, \\
a_{1} \beta_{1}(2)+2 a_{2} \beta_{2}(2) & =q_{1}, \\
a_{1} \beta_{1}(3)+2 a_{2} \beta_{2}(3)+3 a_{3} \beta_{3}(3) & =q_{2}, \\
a_{1} \beta_{1}(4)+2 a_{2} \beta_{2}(4)+3 a_{3} \beta_{3}(4)+4 a_{4} \beta_{4}(4) & =q_{3}, \\
a_{1} \beta_{1}(5)+2 a_{2} \beta_{2}(5)+3 a_{3} \beta_{3}(5)+4 a_{4} \beta_{4}(5)+5 a_{5} \beta_{5}(5) & =q_{4}, \quad \ldots,
\end{aligned}\right.
$$

а ее решение в общем виде дается следующей формулой:

$$
a_{n}=\frac{1}{n !}\left|\begin{array}{cccccc}
\beta_{1}(1) & 0 & 0 & \ldots & 0 & q_{0} \\
\beta_{1}(2) & 2 \beta_{2}(2) & 0 & \ldots & 0 & q_{1} \\
\beta_{1}(3) & 2 \beta_{2}(3) & 3 \beta_{3}(3) & \ldots & 0 & q_{2} \\
\vdots & \vdots & \vdots & \ddots & \vdots & \vdots \\
\beta_{1}(k-1) & 2 \beta_{2}(k-1) & 3 \beta_{3}(k-1) & \ldots & (k-1) \beta_{k-1}(k-1) & q_{k-2} \\
\beta_{1}(k) & 2 \beta_{2}(k) & 3 \beta_{3}(k) & \ldots & (k-1) \beta_{k-1}(k) & q_{k-1}
\end{array}\right| .
$$

Формула (10) полностью решает задачу представления функций мультипликативными агрегатами первого рода.

2. Рассмотрим теперь представление функций мультипликативными агрегатами второго рода. Прологарифмировав и продифференцировав тождество

$$
f(x)=\prod_{n=1}^{\infty}\left(1+x^{n}\right)^{b_{n}},
$$

с учетом (4) получим следующую систему линейных уравнений для определения коэффициентов $b_{n}$ :

$$
\sum_{m \backslash n} m \eta(n, m) a_{m}=q_{n-1},
$$

где $\eta(m)= \pm 1$. Приведем начало этой системы в явном виде:

$$
\left\{\begin{aligned}
-b_{1} & =q_{0}, & -b_{1}-7 b_{7} & =q_{6}, \\
b_{1}-2 b_{2} & =q_{1}, & b_{1}+2 b_{2}+4 b_{4}-8 b_{8} & =q_{7}, \\
-b_{1}-3 b_{3} & =q_{2}, & -b_{1}-3 b_{3}-9 b_{9} & =q_{8}, \\
b_{1}+2 b_{2}-4 b_{4} & =q_{3}, & b_{1}-2 b_{2}+5 b_{5}-10 b_{10} & =q_{9}, \\
-b_{1}-5 b_{5} & =q_{4}, & -b_{1}-11 b_{11} & =q_{10}, \\
b_{1}+2 b_{2}+3 b_{3}-6 b_{6} & =q_{5}, & b_{1}+2 b_{2}+3 b_{3}-4 b_{4}+6 b_{6}-12 b_{12} & =q_{10}, \quad \ldots
\end{aligned}\right.
$$

Функция двух натуральных аргументов $\eta(n, m)$, которая задает распределения знаков в уравнениях системы (12), устроена достаточно сложно; мы не будем здесь описывать ее строение, так как систему (12) целесообразно переписать в другом виде, где будут явно указаны коэффициенты ее уравнений (подобно тому, как систему (5) мы переписали в виде (8)). Для этого вместо $\beta_{m}(n)$ нам понадобятся мигающие функиии второго рода

$$
\gamma_{m}(n)=\left\{\begin{aligned}
1, & \text { если } n=2 k m, \\
-1, & \text { если } n=(2 k+1) m, \\
0, & \text { если } n=h m,
\end{aligned}\right.
$$

при помощи которых систему (12) можно переписать в виде

$$
\sum_{n=1}^{m} n b_{n} \gamma_{n}(m)=q_{m-1} .
$$


Эту систему будем называть определяющей системой для мультипликативных агрегатов второго рода. Начало этой системы имеет вид

$$
\left\{\begin{aligned}
b_{1} \gamma_{1}(1) & =q_{0}, \\
b_{1} \gamma_{1}(2)+2 b_{2} \gamma_{2}(2) & =q_{1}, \\
b_{1} \gamma_{1}(3)+2 b_{2} \gamma_{2}(3)+3 b_{3} \gamma_{3}(3) & =q_{2}, \\
b_{1} \gamma_{1}(4)+2 b_{2} \gamma_{2}(4)+3 b_{3} \gamma_{3}(4)+4 b_{4} \gamma_{4}(4) & =q_{3}, \\
b_{1} \gamma_{1}(5)+2 b_{2} \gamma_{2}(5)+3 b_{3} \gamma_{3}(5)+4 b_{4} \gamma_{4}(5)+5 b_{5} \gamma_{5}(5) & =q_{4}, \quad \ldots,
\end{aligned}\right.
$$

а ее решение в общем виде дается следующей формулой:

$$
a_{n}=\frac{1}{n !}\left|\begin{array}{cccccc}
\gamma_{1}(1) & 0 & 0 & \ldots & 0 & q_{0} \\
\gamma_{1}(2) & 2 \gamma_{2}(2) & 0 & \ldots & 0 & q_{1} \\
\gamma_{1}(3) & 2 \gamma_{2}(3) & 3 \gamma_{3}(3) & \ldots & 0 & q_{2} \\
\vdots & \vdots & \vdots & \ddots & \vdots & \vdots \\
\gamma_{1}(k-1) & 2 \gamma_{2}(k-1) & 3 \gamma_{3}(k-1) & \ldots & (k-1) \gamma_{k-1}(k-1) & q_{k-2} \\
\gamma_{1}(k) & 2 \gamma_{2}(k) & 3 \gamma_{3}(k) & \ldots & (k-1) \gamma_{k-1}(k) & q_{k-1}
\end{array}\right| .
$$

Формула (17) полностью решает задачу представления функций мультипликативными агрегатами первого рода.

3. Применим теперь формулы (10) и (17) для вывода некоторых соотношений теории чисел и комбинаторики.

Например, из представления (7) экспоненты с учетом (10) получаем формулу для вычисления функции Мебиуса:

$$
\mu(n)=(-1)^{n+1}\left|\begin{array}{ccccc}
\beta_{1}(2) & \beta_{2}(2) & 0 & \ldots & 0 \\
\beta_{1}(3) & \beta_{2}(3) & \beta_{3}(3) & \ldots & 0 \\
\vdots & \vdots & \vdots & \ddots & \vdots \\
\beta_{1}(n-1) & \beta_{2}(n-1) & \beta_{3}(n-1) & \ldots & \beta_{n-1}(n-1) \\
\beta_{1}(n) & \beta_{2}(n) & \beta_{3}(n) & \ldots & \beta_{n-1}(n)
\end{array}\right| .
$$

Выведем некоторые формулы для функций $L(n)$. С этой целью введем функцию

$$
H(x)=1+\sum_{n=1}^{\infty} L(n) x^{n}=\prod_{n=1}^{\infty}\left(1-x^{n}\right)^{-1}
$$

и запишем ее логарифмическую производную:

$$
\frac{H^{\prime}(x)}{H(x)}=\sum_{n=1}^{\infty} \sigma(n) x^{n-1} .
$$

Выясним природу коэффициентов $\sigma(n)$. Если эти коэффициенты подставить в систему (8), то получим

$$
\sigma(m)=\sum_{n=1}^{m} n \beta_{n}(m)
$$

откуда видно, что $\sigma(m)$ - это сумма делителей числа $m$.

Разделим теперь фактически $H^{\prime}(x)$ на $H(x)$ :

$$
\frac{L(1)+2 L(2) x+3 L(3) x^{2}+\ldots+n L(n) x^{n-1}+\ldots}{1+L(1) x+L(2) x^{2}+L(3) x^{3}+\ldots+L(n) x^{n}+\ldots}=\sum_{n=1}^{\infty} \sigma(n) x^{n-1},
$$


в результате чего получается система равенств

$$
\left\{\begin{aligned}
\sigma(1) & =L(1), \\
\sigma(1) L(1)+\sigma(2) & =2 L(2), \\
\sigma(1) L(2)+\sigma(2) L(1)+\sigma(3) & =3 L(3), \\
\sigma(1) L(3)+\sigma(2) L(2)+\sigma(3) L(1)+\sigma(4) & =4 L(4), \\
\sigma(1) L(4)+\sigma(2) L(3)+\sigma(3) L(2)+\sigma(4) L(1)+\sigma(5) & =5 L(5), \quad \ldots,
\end{aligned}\right.
$$

т.е. для функции $L(n)$ имеем рекуррентное выражение

$$
(n+1) L(n+1)=\sigma(1) L(n)+\sigma(2) L(n-1)+\ldots+\sigma(n) L(1)+\sigma(n+1) .
$$

Если же систему (23) переписать в виде

$$
\left\{\begin{aligned}
-L(1) & =-\sigma(1), \\
\sigma(1) L(1)-2 L(2) & =-\sigma(2), \\
\sigma(2) L(1)+\sigma(1) L(2)-3 L(3) & =-\sigma(3), \\
\sigma(3) L(1)+\sigma(2) L(2)+\sigma(1) L(3)-4 L(4) & =-\sigma(4), \\
\sigma(4) L(1)+\sigma(3) L(2)+\sigma(2) L(3)+\sigma(1) L(4)-5 L(5) & =-\sigma(5), \quad \ldots,
\end{aligned}\right.
$$

то получим удивительную формулу, устанавливающую связь между суммой делителей натурального числа $n \in \mathbb{N}$ и количеством неупорядоченных представлений в виде суммы равных или неравных натуральных слагаемых этого числа:

$$
L(n)=\frac{(-1)^{n}}{n !}\left|\begin{array}{cccccc}
-1 & 0 & 0 & 0 & \ldots & -\sigma(1) \\
\sigma(1) & -2 & 0 & 0 & \ldots & -\sigma(2) \\
\sigma(2) & \sigma(1) & -3 & 0 & \ldots & -\sigma(3) \\
\vdots & \vdots & \vdots & \vdots & \ddots & \vdots \\
\sigma(n-2) & \sigma(n-3) & \sigma(n-4) & \ldots & -(n-1) & -\sigma(n-1) \\
\sigma(n-1) & \sigma(n-2) & \sigma(n-3) & \ldots & \sigma(1) & -\sigma(n)
\end{array}\right| .
$$

4. Формулы, аналогичные (24) и (26), можно получить и для количества неупорядоченных представлений числа $n \in \mathbb{N}$ в виде суммы неравных натуральных слагаемых. Для этого понадобится ввести в рассмотрение некоторые теоретико-числовые функции. Можно показать (см. [2]), что многие теоретико-числовые функции выражаются через мигающие функции $\beta_{m}(n)$. Например, функцию $\tau(n)$, выражающую количество делителей числа $n$, можно выразить суммой

$$
\tau(n)=\beta_{1}(n)+\beta_{2}(n)+\beta_{3}(n)+\ldots+\beta_{n}(n),
$$

а функция

выражается при $n>2$ произведением

$$
\varepsilon(n)= \begin{cases}1, & \text { если } n \text { простое число, } \\ 0, & \text { если } n \text { составное число, }\end{cases}
$$

$$
\varepsilon(n)=\left(1-\beta_{2}(n)\right)\left(1-\beta_{3}(n)\right) \ldots\left(1-\beta_{n-1}(n)\right),
$$

а формулы (18) и (21) дают представление функций $\mu(n)$ и $\sigma(n)$ через мигающие функции $\beta_{m}(n)$ и т. Д.

Если некоторую теоретико-числовую функцию $\psi(n)$ можно выразить некоторой формулой через мигающие функции, то данной функции можно поставить в соответствие функцию $\tilde{\psi}(n)$, которая получается из $-\psi(n)$ заменой функций $\beta_{m}(n)$ на $\gamma_{m}(n)$, например,

$$
\tilde{\tau}(n)=-\sum_{k=1}^{n} \gamma_{k}(n), \quad \tilde{\sigma}(n)=-\sum_{k=1}^{n} k \gamma_{k}(n), \quad \tilde{\varepsilon}(n)=-\prod_{k=2}^{n-1}\left(1-\gamma_{k}(n)\right)
$$

и т. д. Функции $\tilde{\psi}(n)$ будем называть сопряженными для функций $\psi(n)$. 
Рассмотрим теперь функцию

$$
h(x)=1+\sum_{n=1}^{\infty} l(n) x^{n}=\prod_{n=1}^{\infty}\left(1+x^{n}\right)
$$

и вычислим ее логарифмическую производную, определив коэффициенты ряда Маклорена для $h^{\prime}(x) / h(x)$ из системы $(16)$, с учетом того, что все $b_{k}=1$. В результате получим выражение

$$
\frac{l(1)+2 l(2) x+3 l(3) x^{2}+\ldots+n l(n) x^{n-1}+\ldots}{1+l(1) x+l(2) x^{2}+l(3) x^{3}+\ldots+l(n) x^{n}+\ldots}=\sum_{n=1}^{\infty} \tilde{\sigma}(n) x^{n-1},
$$

откуда вытекает следующая система равенств:

$$
\left\{\begin{aligned}
\tilde{\sigma}(1) & =l(1), \\
\tilde{\sigma}(1) l(1)+\tilde{\sigma}(2) & =2 l(2), \\
\tilde{\sigma}(1) l(2)+\tilde{\sigma}(2) l(1)+\tilde{\sigma}(3) & =3 l(3), \\
\tilde{\sigma}(1) l(3)+\tilde{\sigma}(2) l(2)+\tilde{\sigma}(3) l(1)+\tilde{\sigma}(4) & =4 l(4), \\
\tilde{\sigma}(1) l(4)+\tilde{\sigma}(2) l(3)+\tilde{\sigma}(3) l(2)+\tilde{\sigma}(4) l(1)+\tilde{\sigma}(5) & =5 l(5), \quad \ldots,
\end{aligned}\right.
$$

т.е. для функции $l(n)$ имеем рекуррентное выражение

$$
(n+1) l(n+1)=\tilde{\sigma}(1) l(n)+\tilde{\sigma}(2) l(n-1)+\ldots+\tilde{\sigma}(n) l(1)+\tilde{\sigma}(n+1) .
$$

Если же систему (29) переписать в виде

$$
\left\{\begin{aligned}
-l(1) & =-\tilde{\sigma}(1), \\
\tilde{\sigma}(1) l(1)-2 l(2) & =-\tilde{\sigma}(2), \\
\tilde{\sigma}(2) l(1)+\tilde{\sigma}(1) l(2)-3 l(3) & =-\tilde{\sigma}(3), \\
\tilde{\sigma}(3) l(1)+\tilde{\sigma}(2) l(2)+\tilde{\sigma}(1) l(3)-4 l(4) & =-\tilde{\sigma}(4), \\
\tilde{\sigma}(4) l(1)+\tilde{\sigma}(3) l(2)+\tilde{\sigma}(2) l(3)+\tilde{\sigma}(1) l(4)-5 l(5) & =-\tilde{\sigma}(5), \quad \ldots,
\end{aligned}\right.
$$

то получим следующую формулу для вычисления количества неупорядоченных представлений в виде суммы неравных натуральных слагаемых числа $n \in \mathbb{N}$ :

$$
l(n)=\frac{(-1)^{n}}{n !}\left|\begin{array}{cccccc}
-1 & 0 & 0 & 0 & \ldots & -\tilde{\sigma}(1) \\
\tilde{\sigma}(1) & -2 & 0 & 0 & \ldots & -\tilde{\sigma}(2) \\
\tilde{\sigma}(2) & \tilde{\sigma}(1) & -3 & 0 & \ldots & -\tilde{\sigma}(3) \\
\vdots & \vdots & \vdots & \vdots & \ddots & \vdots \\
\tilde{\sigma}(n-2) & \tilde{\sigma}(n-3) & \tilde{\sigma}(n-4) & \ldots & -(n-1) & -\tilde{\sigma}(n-1) \\
\tilde{\sigma}(n-1) & \tilde{\sigma}(n-2) & \tilde{\sigma}(n-3) & \ldots & \tilde{\sigma}(1) & -\tilde{\sigma}(n)
\end{array}\right| .
$$

Таким образом, формулы (30) и (32) для вычисления арифметической функции $l(n)$ получаются из формул $(24)$ и (26) для функции $L(n)$ простой заменой функций $\sigma(n)$ на сопряженные им функции $\tilde{\sigma}(n)$.

5. В заключение отметим, что хотя мигающие функции $\beta_{m}(n)$ и $\gamma_{m}(n)$ были определены вербально, для них существуют простые аналитические выражения. Если ввести в рассмотрение величины

$$
\alpha_{m}=\cos (2 \pi / m)+i \sin (2 \pi / m) \equiv \sqrt[m]{1},
$$

то для мигающих функций $\beta_{m}(n)$ и $\gamma_{m}(n)$ будут справедливы следующие формулы:

$$
\begin{gathered}
\beta_{m}(n)=\frac{1+\alpha_{m}^{n}+\alpha_{m}^{2 n}+\ldots+\alpha_{m}^{(m-1) n}}{m}, \\
\gamma_{m}(n)=\frac{\alpha_{2 m}^{n}\left(1+\alpha_{m}^{n}+\alpha_{m}^{2 n}+\ldots+\alpha_{m}^{(m-1) n}\right)}{m},
\end{gathered}
$$

и, в частности, $\beta_{1}(n) \equiv 1, \gamma_{1}(n)=(-1)^{n}$. 
Таким образом, различные арифметические функции, сконструированные из мигающих функций $\beta_{m}(n)$ и $\gamma_{m}(n)$, получают аналитические выражения через тригонометрические функции. Это позволяет делать оценки роста арифметических функций, исследовать их асимптотики, аппроксимировать функции натурального аргумента непрерывными и гладкими функциями вещественного и комплексного аргумента и т. д. Впрочем, все эти вопросы далеко выходят за рамки данной статьи.

Заметим еще, что с бесконечными произведениями степенных биномов и с приложениями мигающих функций можно более подробно познакомиться по монографии авторов «Мемуар о бесконечных произведениях» (см. [1]) и по учебному пособию «Введение в специальность. Конспект лекций» (см. [2]), ставшему, впрочем, библиографической редкостью после чеченской войны 19941996 гг.

\section{СПИСОК ЛИТЕРАТУРЫ}

1. Бурлаков В. М., Бурлаков М. П. Мемуар о бесконечных произведениях. - М.: Изд-во Л. А. Ким, 2019.

2. Бурлаков М. П. Введение в специальность. Конспект лекций. - Грозный: Изд-во Чечено-Ингуш. ун-та, 1985.

3. Виноградов И. М. Основы теории чисел. - М.: Наука, 1972.

4. Меньшиков М. В., Ревякин А. М., Копылова А. Н., Макаров Ю. Н., Стечкин Б. С. Комбинаторный анализ. Задачи и упражнения. - М.: Наука, 1982.

5. Эйлер Л. Введение в анализ бесконечных. - М.: ГИФМЛ, 1961.

Бурлаков Михаил Петрович

Тверской государственный университет

E-mail: burlakovmihail@mail.ru

Бурлаков Валерий Михайлович

Пензенский государственный университет 